\title{
O EFEITO DA TURBULÊNCIA TECNOLÓGICA NO SUCESSO DE NOVOS PRODUTOS
}

\author{
THE EFFECT OF \\ TECHNOLOGICAL TURBULENCE \\ ON NEW PRODUCTS SUCCESS
}

Recebido em: 30/05/2016 Aceito em: 04/07/2016

mirelajs@unisc.br

\author{
Mirela Jeffman dos Santos ${ }^{1}$ \\ Marcelo Gattermann Perin ${ }^{2}$ \\ Cláudio Hoffmann Sampaio ${ }^{2}$
}

Resumo: O estudo objetivou analisar a influência das orientações ao consumidor (OC), à aprendizagem $(\mathrm{OA})$ e à inovação $(\mathrm{OI})$ na habilidade de desenvolvimento de novos produtos (HDNP), e desta no sucesso de novos produtos (SNP), bem como a influência da turbulência tecnológica (TT) na relação entre a HDNP e o SNP. Por meio de levantamento realizado com empresas industriais brasileiras, verificou-se efeito positivo das três orientações estratégicas (ao consumidor, à aprendizagem e à inovação) na habilidade de desenvolvimento de novos produtos, sendo que a orientação à inovação revelou-se o antecedente de maior efeito. Constatou-se também que a turbulência tecnológica modera a relação entre a habilidade e o sucesso de novos produtos.

Palavras-chave: Orientação ao Consumidor. Orientação à Aprendizagem. Orientação à Inovação. Sucesso de Novos Produtos. Turbulência Tecnológica.

Abstract: The paper aimed to analyze the influence of consumer (CO), learning (LO) and innovation orientations (IO) on new products development ability (NPDA), as well as its impact on the success of new products (SNP). In addition, its objective was also to analyze the technological turbulence in the relationship between NPDA and SNP. Through a survey done with industrial companies from Brazil, it was found a positive effect of three strategic orientations (consumer, learning and innovation) on new products development ability, as well as innovation orientation was the most influent antecedent on NPDA. Furthermore, the research pointed out technological turbulence moderates the relationship between ability and success of new products.

Keywords: Consumer Orientation. Learning Orientation. Innovation Orientation. New Products Success. Technological Turbulence.

\footnotetext{
${ }^{1}$ Universidade de Santa Cruz do Sul - UNISC - Santa Cruz do Sul - Rio Grande do Sul - Brasil.

${ }^{2}$ Pontifícia Universidade Católica do Rio Grande do Sul - PUCRS - Porto Alegre - Rio Grande do Sul - Brasil.
} 


\section{INTRODUÇÃO}

Em um mercado no qual preponderam incertezas e rápidas mudanças tecnológicas, procurar identificar os recursos empresariais que levam a um desempenho diferenciado e, consequentemente, à vantagem competitiva, têm sido um dos maiores interesses acadêmicos desde a década passada (WEI; MORGAN, 2004).

Diversos estudos (ver, por exemplo, REID; BREMTANI, 2010) têm questionado por que determinadas empresas conseguem alcançar altos níveis de sucesso enquanto outras não e o quê propicia que as empresas tomem decisões estratégicas corretas e outras não (TALKE; HULTINK, 2010). Um dos fatores que tem sido apontado como primordial para a sobrevivência e competitividade no mercado é a postura inovadora (AKMAN; YILMAZ, 2008; STOCK; ZACHARIAS, 2011).

Neste contexto, a inovação de produto - que é definida como o processo de trazer ao uso do mercado novos produtos e/ou novas tecnologias (LUKAS; FERRELL, 2000) - assume papel destacado no desempenho global da organização (LANGERAK, HULTINK; ROBBEN, 2004; LEDWITH; O'DWYER, 2009). O sucesso do lançamento de novos produtos no mercado tem recebido significativa atenção em estudos internacionais (por exemplo, KLEINSCHMIDT, BRENTANI; SALOMO, 2007; LEENDERS, ENGELEN; KRATZER, 2007; TALKE; HULTINK, 2010; NAKADA; IM, 2010; REID; BREMTANI, 2010), que têm revelado um conjunto de condicionantes significativos para o seu alcance.

Nesta linha, merecem destaque os fatores internos à organização que envolvem aspectos relacionados à cultura organizacional e são fortes impulsionadores da postura de inovação (VAN DE VEN, 1986). Estes são particularmente estudados pela Visão Baseada em Recursos (RBV), que é referência na literatura de marketing estratégico quando se trata dos recursos organizacionais que conduzem a uma posição destacada no mercado e à performance superior (KOZLENKOVA, SAMAHA; PALMATIER, 2013).

A literatura apresenta um conjunto de orientações estratégias que conduzem ao sucesso de novos produtos, como orientação ao consumidor (OC) (NARVER, SLATER; MaCLACHLAN, 2004; AUGUSTO; COELHO, 2009), a orientação à aprendizagem (OA) (CALANTONE, CAVUSGILA; ZHAO, 2002; RHEE, PARK E LEE, 2010) e a orientação à inovação (OI) (KLEINSCHMIDT, BRENTANI; SALOMO, 2007; SALOMO, TALKE; STRECKER, 2008) que são construtos amplamente testados nos mais diversos contextos.

Orientação ao Consumidor significa a postura organizacional direcionada à busca, obtenção e aproveitamento de informações oriundas dos consumidores (ATUAHENE-GIMA, 2005).

Orientação à Aprendizagem refere-se à postura organizacional voltada para a construção e incorporação de conhecimento a partir de experiências próprias ou de outras empresas atuantes no mercado (HULT, HURLEY; KNIGHT, 2004).

Orientação à Inovação refere-se à cultura organizacional que prioriza o surgimento de novas ideias, a quebra de paradigmas e a criatividade dos colaboradores, enfatizando a importância da criação de novos produtos para o sucesso da organização (KLEINSCHMIDT, BRENTANI; SALOMO, 2007).

Estudos do CEPE [ISSN 1982-6729]. Santa Cruz do Sul, n. 43, p. 119-135, jan./jun. 2016. https://online.unisc.br/seer/index.php/cepe/index 
Estes construtos, no entanto, têm sido apontados como antecedentes diretos do sucesso de novos produtos (SNP) (por exemplo, no estudo de TALKE, SALOMO; KOCK, 2011) ou mediados pelo compartilhamento de conhecimento (como no estudo de LI; CALANTONE, 1998), pela vantagem do produto ou pelas atividades anteriores ao lançamento do novo produto (LANGERAK, HULTINK; ROBBEN, 2004) e ainda alguns autores têm apontado relações entre os construtos (como, por exemplo, RHEE, PARK, LEE, 2009; HULT, HURLEY; KNIGHT, 2004). Assim, a literatura tem negligenciado uma etapa anterior ao SNP que se refere à habilidade de desenvolvimento de novos produtos (HDNP). Alguns estudos têm se aproximado deste construto quando mencionam a criatividade e a velocidade para a solução de problemas (WEI; ATUAHENE-GIMA, 2009) e a sinergia técnica (CALANTONE, CHAN; CUI, 2006), entretanto em alguns casos a habilidade é apresentada de forma muito similar a outros conceitos como a orientação ao consumidor quando refere-se à capacidade de utilização de informações (CITRIN, LEE; McCULLOUGH, 2007) ou a orientação à aprendizagem quando refere-se à retenção e interpretação de conhecimento (MARSH; STOCK, 2006) ou ainda orientação à inovação quando refere-se aos recursos de inovação (SNOJ, MILFELNER; GABRIJAN, 2007). Desta forma, o conceito de habilidade de desenvolvimento de novos produtos revela-se pouco claro na literatura, constatando-se assim carência de indicadores para o teste do construto. Uma das exceções é o estudo de Hooley et al (2005) que nomeia o construto de "capacidade de inovação em marketing" e apresenta a habilidade de lançamento do novo produto e a eficiência no seu processo de desenvolvimento como indicadores.

Constata-se assim a escassez de estudos que tenham testado o impacto dos condicionantes internos na habilidade de desenvolvimento de novos produtos, bem como desta no sucesso de novos produtos. Além disso, os estudos também têm apontado a influência do ambiente externo na forma em que as organizações atuam (CALANTONE, GARCIA; DROGE, 2003; KUIVALAINEN ET AL., 2004; ATUAHENE-GIMA; WEI, 2010), especialmente a Turbulência Tecnológica (TT) (CARBONELL, RODRIGUEZ-ESCUDERO; PUJARI, 2009). A TT refere-se à velocidade e à frequência em que novas tecnologias surgem no setor em que a empresa atua, tornando as tecnologias anteriores obsoletas (ZHOU, YIM; TSE, 2005) e pouco se sabe sobre como as organizações inovadoras operam diante de condições turbulentas do ambiente externo (HULT, HURLEY; KNIGHT, 2004).

Diante desta lacuna identificada na literatura, o presente estudo tem por objetivo analisar a influência das orientações ao consumidor $(\mathrm{OC})$, à aprendizagem $(\mathrm{OA})$ e à inovação $(\mathrm{OI})$ na habilidade de desenvolvimento de novos produtos (HDNP), bem como desta no sucesso de novos produtos (SNP). O estudo visa ainda analisar a influência da turbulência tecnológica (TT) na relação entre a HDNP e s SNP.

O presente estudo está dividido em cinco partes, incluída esta introdução. A seguir apresentase a teoria que deu suporte à construção do modelo teórico que foi testado neste estudo. Após, apresentam-se os procedimentos metodológicos realizados na pesquisa de campo. Em seguida, os resultados são apresentados e analisados. Por fim, as conclusões e implicações da pesquisa são expostas.

\section{MODELO TEÓRICO}

Estudos do CEPE [ISSN 1982-6729]. Santa Cruz do Sul, n. 43, p. 119-135, jan./jun. 2016. https://online.unisc.br/seer/index.php/cepe/index 
A orientação para o consumidor $(O C)$ refere-se à produção e uso de informações a respeito das necessidades atuais e futuras dos clientes (ATUAHENE-GIMA, 2005). As necessidades atuais, também chamadas de expressas, são aquelas que o consumidor possui consciência e por isso está apto a expressá-las. Já as necessidades futuras ou latentes não se encontram no nível de consciência do consumidor e por isso este não está apto a comunicá-las de forma explícita (NARVER, SLATER; MacLACHLAN, 2004).

A OC é parte da cultura corporativa e abarca um conjunto de valores e crenças que reforçam o foco que a organização possui no consumidor (DESHPANDÉ, FARLEY; WEBSTER JR., 1993). Neste sentido, a postura orientada para o consumidor envolve a proximidade da organização com o consumidor (MACINTOSH, 2007) e a captação de seus feedbacks com o intuito de compreender mais profundamente os seus comportamentos, as suas preferências e as suas opiniões (AUH; MENGUC, 2005).

Deste modo, a OC não contempla apenas a captação de informações dos consumidores, envolve também o entendimento das suas necessidades e a análise detalhada de como a organização poderá satisfazê-las (AUGUSTO; COELHO, 2009). Por meio da OC, a organização dispõe de uma fonte de ideias para mudanças, melhorias nos seus produtos atuais $\mathrm{e}$ desenvolvimento de novos produtos (NGO; O'CASS, 2012; SPANJOL, QUALLS E ROSA, 2011).

Neste sentido, a orientação ao consumidor contribui para a inovação de produto, pois promove maior conhecimento sobre comportamentos de compra e mercado potencial (WEl; MORGAN, 2004), permite que a empresa aproveite as oportunidades do mercado para promover inovações que sejam completamente alinhadas às necessidades dos clientes (LI; CALANTONE, 1998) e capacita a organização a desenvolver produtos com maior vantagem perante os concorrentes (LANGERAK, HULTINK; ROBBEN, 2004). Diante do exposto, apresenta-se a seguinte hipótese:

H1: A orientação ao consumidor possui impacto positivo na habilidade de desenvolvimento de novos produtos.

A orientação para a aprendizagem refere-se à atividade da organização de criar e utilizar conhecimento com o intuito de conquistar vantagem competitiva. Esta prática engloba as atividades de captar informações referentes ao comportamento dos clientes, às transformações de mercado e as ações dos concorrentes (CALANTONE, CAVUSGILA; ZHAO, 2002) e incorporá-las às práticas de gestão de modo a adotar novos comportamentos, valores e crenças (HULT, HURLEY; KNIGHT, 2004).

Organizações voltadas para a aprendizagem adotam um estilo de comportamento que estimula o surgimento de novas ideias e a execução de mudanças, permitindo que os colaboradores questionem a maneira tradicional de desempenhar as tarefas e possam ousar nas suas atividades profissionais (ATUAHENE-GIMA, SLATER; OLSON, 2005). Neste sentido, Baker e Sinkula (2007) ressaltam os benefícios da aprendizagem criativa, que se desprende de modelos mentais preexistentes e conduz a novas formas de pensar baseadas no rompimento de antigos paradigmas. 
A orientação para a aprendizagem ocorre primeiramente em nível da cultura da organização (HULT, HURLEY; KNIGHT, 2004), que deve valorizar a aprendizagem, estimular o comprometimento de seus colaboradores com esta atividade, promover a socialização do conhecimento internamente, compartilhar a sua visão e objetivos estratégicos em todos os níveis institucionais, bem como formar uma equipe de profissionais que esteja aberta para novas ideias e aceite maneiras diferentes de desempenhar as atividades (CALANTONE, CAVUSGILA; ZHAO, 2002).

Uma empresa orientada à aprendizagem possui sua capacidade de inovação incrementada, pois se torna mais capacitada para aproveitar as oportunidades do mercado decorrentes de novas necessidades dos consumidores, estuda as forças e fraquezas dos seus concorrentes e aprende a partir dos sucessos e fracassos dos mesmos e ainda está mais apta a descobrir e implementar novas tecnologias, rompendo antigos paradigmas e mostrando-se aberta a novas ferramentas que podem agilizar os processos (CALANTONE, CAVUSGILA; ZHAO, 2002). Assim, o grau em que uma empresa é orientada para a aprendizagem e que consegue explorar, obter e compartilhar novos conhecimentos de forma descentralizada influencia o seu grau de inovação (RHEE, PARK; LEE, 2010).

Deste modo, as organizações orientadas à aprendizagem estimulam os seus colaboradores a atuar como empreendedores, a questionar a maneira tradicional de desempenhar as tarefas e a assumir riscos, tolerando a ocorrência de erros sem adotar medidas punitivas. Esse tipo de ambiente favorece o desenvolvimento de habilidades para desenvolver novos produtos, pois oferece maior autonomia aos profissionais e mais liberdade para expressarem suas ideias e para ousarem no desempenho das suas atividades (BRENTANI; KLEINSCHMIDT, 2004; ATUAHENE-GIMA, SLATER; OLSON, 2005; KLEINSCHMIDT, BRENTANI; SALOMO, 2007; BARCZAK, SULTAN; HULTINK, 2007).

A OA tem sido apontada como antecedente da habilidade de desenvolvimento de novos produtos e foi confirmada nos estudos de Calantone, Cavusgila e Zhao (2002), Hult, Hurley e Knight (2004) e Rhee, Park e Lee (2010). Diante do exposto, apresenta-se a seguinte hipótese:

H2: A orientação à aprendizagem possui impacto positivo na habilidade de desenvolvimento de novos produtos.

A orientação à inovação refere-se a um aspecto proveniente da cultura organizacional que facilita a implementação da inovação (HURLEY; HULT, 1998). A estrutura da orientação à inovação compreende a filosofia de aprendizagem, o direcionamento estratégico e as crenças transfuncionais que direcionam as estratégias, ações, comportamentos, competências e processos organizacionais para promover o pensamento inovador e facilitar o desenvolvimento e a execução de inovações de sucesso (SIGUAW, SIMPSON; ENZ, 2006).

A cultura inovativa refere-se ao ambiente organizacional que favorece o surgimento da inovação por meio do estímulo ao empreendedorismo, da abertura a novas ideias, do encorajamento dos colaboradores a assumir os riscos ao longo do processo (BRENTANI; KLEINSCHMIDT, 2004) e da valorização da atividade de desenvolvimento de novos produtos para o sucesso da organização (KLEINSCHMIDT, BRENTANI; SALOMO, 2007). 
O nível de abertura à inovação de uma organização envolve o quanto os seus colaboradores são receptivos para considerar a adoção de uma inovação (HULT, HURLEY; KNIGHT, 2004). Neste sentido, Van de Ven (1986) aponta alguns desafios a serem superados quando da implantação da inovação, entre eles o gerenciamento de ideias, a resistência à mudança, a tendência a desempenhar atividades rotineiras e a dificuldade para trabalhar em equipe.

Organizações orientadas à inovação enfatizam a aprendizagem, estimulam a tomada de decisão participativa e proporcionam a comunicação aberta entre as diversas áreas funcionais (HURLEY; HULT, 1998), o que as tornam mais capacitadas para experimentar novas ideias, formando assim um ambiente de estímulo à criatividade - que consiste em uma atividade chave da inovação (BARCZAK, SULTAN; HULTINK, 2007). Assim, a orientação à inovação reflete os valores e crenças que guiam a atividade de inovação da organização (TALKE, SALOMO; KOCK, 2011).

A orientação à inovação tem sido apontada como antecedente da capacidade e do sucesso da inovação (como por exemplo, nos estudos de KLEINSCHMIDT, BRENTANI; SALOMO, 2007; SALOMO, TALKE; STRECKER, 2008; TALKE, SALOMO; KOCK, 2011). Diante do exposto, apresenta-se a seguinte hipótese:

H3: A orientação à inovação possui impacto positivo na habilidade de desenvolvimento de novos produtos.

A inovação de produto consiste em um processo interativo que se inicia pela identificação de uma oportunidade (um novo mercado e/ou um novo produto) que leva ao desenvolvimento, à produção e à elaboração do marketing direcionado ao novo produto com vistas a alcançar um desempenho destacado no mercado (GARCIA; CALANTONE, 2002). A habilidade de desenvolvimento de novos produtos (HDNP) refere-se à habilidade da organização para gerar, criar ou desenvolver inovações de produto (SUBRAMANIAM, YOUNDT, 2005; MENGUC; AUH, 2009).

Vorhies, Morgan e Autry (2009) apresentam a HDNP como um conjunto de habilidades construídas por meio da integração do conhecimento especializado dos colaboradores da organização que permitem o emprego de recursos para alcançar os objetivos da inovação de produto.

A organização com alta HDNP possui maior capacidade para promover inovações que reflitam sugestões e demandas oriundas do ambiente externo (AKMAN; YILMAZ, 2008), tonando-se mais hábil para criar e lançar produtos de sucesso no mercado, o que resulta em índices mais altos de satisfação e lealdade do consumidor (HOOLEY et al, 2005).

Assim, a HDNP contribui para o sucesso do novo produto, pois a satisfação e lealdade do consumidor resultam no incremento do volume de vendas e na conquista de maior fatia de mercado (HOOLEY et al, 2005). Diante do exposto, apresenta-se a seguinte hipótese:

H4: A habilidade de desenvolvimento de novos produtos possui impacto positivo no sucesso de novos produtos.

A turbulência do ambiente existe quando a frequência e a imprevisibilidade das mudanças do mercado e/ou tecnológicas agravam o risco e a incerteza do planejamento estratégico do processo de DNP (CALANTONE, GARCIA; DROGE, 2003). A Turbulência Tecnológica (TT) refere-se ao aumento 
nas taxas de crescimento das inovações tecnológicas no setor econômico em que a empresa atua, provocando impacto no desenvolvimento interno de suas inovações. Há duas variáveis que influenciam a intensidade de TT de um ambiente: (I) rapidez - que se refere à frequência das mudanças no ambiente, incluindo, por exemplo, a velocidade de inovação nas tecnologias e (II) imprevisibilidade - que se refere ao quanto as mudanças são passíveis de se antever (BUGANZA, DELL'ERA; VERGANI, 2009). A TT possui

Quando as mudanças no ambiente tecnológico são velozes e imprevisíveis, o conhecimento existente em uma organização pode tornar-se ultrapassado rapidamente. Empresas que reconhecem que operam em um ambiente turbulento e conseguem responder a ele prontamente são melhor capacitadas para criar e aplicar novos conhecimentos. Em oposição, empresas que atuam em ambientes menos turbulentos tornam-se mais complacentes e não aprendem tão rapidamente a partir do mercado (DROGE, CLAYCOMB; GERMAIN, 2003).

Os ambientes instáveis tecnologicamente apresentam risco e incerteza nos processos de desenvolvimento de inovações, pois as previsões tornam-se mais difíceis, os produtos existentes tornam-se obsoletos em um período de tempo menor (RIJSDIJK, LANGERAK; HULTINK, 2010), os concorrentes são mais agressivos, as oportunidades de mercado podem ser reduzidas e os riscos do negócio são maiores (CALANTONE, GARCIA; DROGE, 2003).

As condições turbulentas requerem que as equipes contribuam de forma expressiva e sejam mais comprometidas e dedicadas no desempenho dos seus papéis durante o desenvolvimento de inovações de produto (DAYAN; ELBANNA, 2011). Assim, os profissionais precisam ajustar suas ações, práticas e crenças e adaptar-se mais rapidamente às mudanças (AKGÜN, KESKIN; BYRNE, 2012).

Neste sentido, quando o ambiente é tecnologicamente turbulento a HDNP torna-se mais importante para o alcance do sucesso de novos produtos, posto que é essencial que a organização procure reduzir o tempo de desenvolvimento dos seus projetos de inovação e minimizar os riscos e incertezas a eles associados (CALANTONE, GARCIA; DROGE, 2003). Diante do exposto, a seguinte hipótese é apresentada:

H5: A relação positiva entre a habilidade de desenvolvimento de novos produtos e o sucesso de novos produtos é fortalecida quando a turbulência tecnológica é maior.

A Figura 1 apresenta o modelo teórico a ser testado neste estudo.

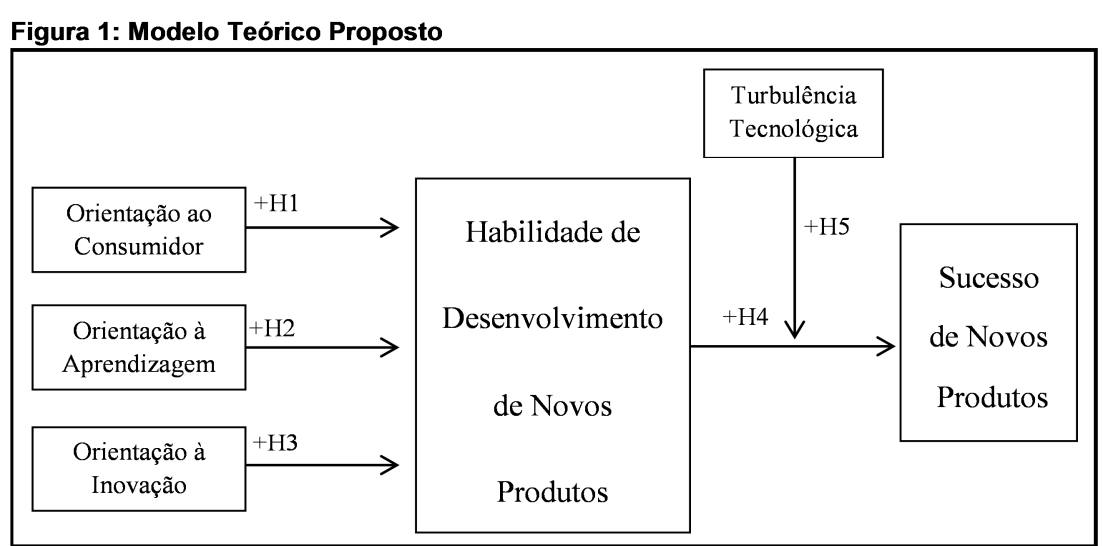

Fonte: elaborado pelos pesquisadores

Estudos do CEPE [ISSN 1982-6729]. Santa Cruz do Sul, n. 43, p. 119-135, jan./jun. 2016. https://online.unisc.br/seer/index.php/cepe/index 


\section{MÉTODO}

\subsection{Procedimentos de Coleta de Dados e Amostragem}

No presente estudo, um grupo de empresas brasileiras com mais de 30 colaboradores foi definido como a população alvo. Estas empresas estão localizadas em diferentes regiões do país. E, de maneira semelhante a outros estudos sobre a mesma temática (como, por exemplo, JAWORSKI; KOHLI, 1993; SINKULA ET AL 1997; BAKER; SINKULA, 2007), a pesquisa englobou empresas industriais.

O questionário foi originalmente escrito em inglês e, posteriormente traduzido para o português seguindo o procedimento da tradução reversa (DILLON, MADDEN; FIRTLE, 1994). O questionário estruturado foi enviado por e-mail aos CEOs de 3.000 companhias do ramo industrial no Brasil. Os CEOs foram definidos como a população mais adequada para participar da pesquisa por possuírem responsabilidade direta no processo de tomada de decisão das empresas.

Por meio deste procedimento, foram obtidos 273 questionários válidos, o que corresponde a 9,1\% de taxa de resposta. Este resultado constitui uma taxa aceitável (MENON ET AL 1996) e é considerado alto se comparado a outros estudos realizados em países em desenvolvimento (por exemplo, ZOU ET AL 1997).

Para controlar vieses de não respondentes, os questionários foram divididos em dois grupos (os primeiros e os últimos respondidos) e testados por meio do teste t com o intuito de comparar diferenças entre os grupos. No entanto, o teste não indicou diferenças significativas entre os grupos para todas as variáveis.

\section{Medidas}

Os construtos usados nesta pesquisa foram desenvolvidos com base na literatura, especificamente em Hooley et al (2005). O construto Orientação ao Consumidor foi baseado em Narver e Slater (1990), aplicado com uma escala Likert de 7 pontos. A Orientação à Aprendizagem foi conceituada conforme a definição de Sinkula et al (1997) e aplicada com uma escala do tipo Likert de 5 pontos. A Orientação à Inovação foi mensurada como a tendência da empresa de iniciar procedimentos novos e engajar-se em mais processos de inovação com vistas a alcançar seu público-alvo (WEST; ANDERSON, 1996).

A Habilidade de Desenvolvimento de Novos Produtos foi desenvolvida a partir das proposições de Hooley, Möller e Broderick (1998) e Day (1994), utilizando uma escala do tipo Likert de 5 pontos. A Turbulência Tecnológica foi mensurada com indicador baseado em Hooley et al (2005). Finalmente, o Sucesso de Novos Produtos foi mensurado com base nos indicadores de Baker e Sinkula (1999).

Também foram incluídas condições ambientais (velocidade de mudança de desejos e necessidades do mercado e intensidade da concorrência), além do tamanho da empresa (em número de funcionários), como variáveis de controle. Pesquisas anteriores mostram que estas variáveis têm um impacto positivo no Sucesso de Novos Produtos (ATUAHENE-GIMA, 2005). Ambas as variáveis 
do ambiente foram mensuradas com uma escala Likert de 5 pontos. A Tabela 1 descreve a confiabilidade e validade das medidas.

Para validar as medidas, primeiramente foram analisados possíveis vieses (PODSAKOFF ET $A L$ 2003). O teste de fator único de Harman foi executado com o intuito de checar variância de método comum (Podsakoff et al., 2003). De acordo com este teste, a variância de método comum seria um problema se um fator único em uma análise fatorial sem rotação contasse para a maioria da covariância entre as variáveis.

A análise fatorial exploratória de todos os construtos multi-item neste estudo revelou cinco fatores com Eigen Values maiores do que um. O primeiro fator estimou 33,78\% da variância nos dados. Todos os fatores explicaram $70,132 \%$ da variância nas variáveis originais. $E$ ainda, os efeitos do fator do método latente único não mensurado foram controlados no modelo estrutural (PODSAKOFF ET AL 2003). Os resultados demonstraram que a variância de método comum não enviesou as medidas.

Tabela 1 - Confiabilidade e Validade das Medidas

\begin{tabular}{|c|c|c|c|c|c|c|c|c|}
\hline \multirow{2}{*}{ Item } & \multirow{2}{*}{ AVE } & \multirow{2}{*}{ CR } & \multirow{2}{*}{$\alpha$} & \multicolumn{5}{|c|}{ Correlações entre os construtos } \\
\hline & & & & 1 & 2 & 3 & 4 & 5 \\
\hline 1. Orientacão ao Consumidor & 0.51 & 0.84 & 0.83 & 0,71 & & & & \\
\hline 2. Orientação à Aprendizagem & 0.56 & 0.83 & 0.82 & $0,518^{* *}$ & 0,75 & & & \\
\hline 3. Orientação à Inovação & 0.67 & 0.89 & 0.89 & $0,348^{\star *}$ & $0,419^{* *}$ & 0,82 & & \\
\hline $\begin{array}{l}\text { 4. Habilidade de } \\
\text { Desenvolvimento de Novos } \\
\text { Produtos }\end{array}$ & 0.51 & 0.76 & 0.75 & $0,377^{\star *}$ & $0,386^{\star *}$ & $0,509^{\star *}$ & 0,71 & \\
\hline 5. Sucesso de Novos Produtos & 0.71 & 0.88 & 0.87 & $0,242^{* *}$ & $0,146^{*}$ & $0,287^{* *}$ & $0,474^{* *}$ & 0,84 \\
\hline 6. Turbulência Tecnológica & - & - & - & 0,036 & 0,051 & 0,027 & $-0,017$ & 0,063 \\
\hline
\end{tabular}

Nota: Os elementos na diagonal (em negrito) são a raiz quadrada da Variância Extraída Média (AVE).

${ }^{*} p<0,05 \mid{ }^{* *} p<0,01$

Fonte: dados da pesquisa

Para testar a validade convergente, foi conduzida a análise fatorial confirmatória do modelo testado. O modelo apresentou índices considerados altamente aceitáveis ( $X 2=245,466, d f=141$, $\mathrm{X} 2 / \mathrm{df}=1,741, \mathrm{RMSEA}=0,052, \mathrm{SRMR}=0,060, \mathrm{TLI}=0,952$, e CFI $=0,960$ ).

A Tabela 2 apresenta a média, o desvio padrão, as cargas fatoriais e os valores $t$ obtidos para todos os construtos investigados neste estudo. As cargas de todos os itens em cada construto revelaram-se altamente significativos $(p<0,001)$ e superaram o valor mínimo sugerido de 0,6 sugerido por Hulland (1999). Os valores altos dos coeficientes alfa, a variância extraída média e os índices de confiabilidade de cada construto foram reveladores de unidimensionalidade.

As cargas fatoriais, os valores $t$ e a confiabilidade composta expressaram altos níveis de validade convergente. Para avaliar a validade discriminante, foram contrastadas a correlação de cada par de fatores com a raiz quadrada da variância extraída de cada fator (FORNELL; LARCKER, 1981). Em cada caso, a variância média extraída superou a correlação quadrada, sustentando a validade discriminante. Em resumo, foram constatadas evidência de unidimensionalidade, validade convergente, validade discriminante, e invariância medida para o modelo testado. Todos os construtos foram validados para testes subsequentes. 
Tabela 2 - Média, Desvio Padrão, Carga Fatorial e Valores $t$

\begin{tabular}{|c|c|c|c|c|}
\hline Item & Média & D.P. & $\begin{array}{c}\text { Carga } \\
\text { Fatorial }\end{array}$ & $\begin{array}{l}\text { Valore } \\
\text { s } t\end{array}$ \\
\hline \multicolumn{5}{|l|}{ Orientação ao Consumidor } \\
\hline $\begin{array}{l}\text { Nossos objetivos e estratégias são guiados pela } \\
\text { criação de satisfação ao cliente. }\end{array}$ & 5,80 & 1,12 & 0,672 & \\
\hline $\begin{array}{l}\text { As estratégias competitivas são baseadas na } \\
\text { compreensão das necessidades dos clientes. }\end{array}$ & 5,52 & 1,24 & 0,848 & 11,493 \\
\hline $\begin{array}{l}\text { As funções do negócio estão integradas para servir às } \\
\text { necessidades do mercado. }\end{array}$ & 5,45 & 1,17 & 0,743 & 10,496 \\
\hline $\begin{array}{l}\text { As estratégias do negócio são guiadas pelo incremento } \\
\text { de valor para os clientes. }\end{array}$ & 5,37 & 1,26 & 0,671 & 9,636 \\
\hline $\begin{array}{l}\text { Nossos administradores compreendem como os } \\
\text { empregados podem contribuir para oferecer valor para } \\
\text { os clientes. }\end{array}$ & 5,27 & 1,36 & 0,611 & 8,872 \\
\hline \multicolumn{5}{|l|}{ Orientação à Aprendizagem } \\
\hline $\begin{array}{l}\text { Os administradores concordam que a habilidade de } \\
\text { nossa empresa em aprender é a chave para a } \\
\text { vantagem competitiva. }\end{array}$ & 4,11 & 0,88 & 0,642 & \\
\hline $\begin{array}{l}\text { O treinamento e a aprendizagem dos empregados são } \\
\text { vistos como um investimento ao invés de despesa. }\end{array}$ & 4,30 & 0,86 & 0,788 & 10,591 \\
\hline $\begin{array}{l}\text { Os valores básicos de nossa empresa incluem o } \\
\text { aprendizado como um fator chave para melhorias. }\end{array}$ & 4,30 & 0,82 & 0,891 & 11,209 \\
\hline $\begin{array}{l}\text { Nossos funcionários entendem que nossas percepções } \\
\text { sobre o mercado devem ser continuamente } \\
\text { questionadas. }\end{array}$ & 3,60 & 0,96 & 0,643 & 9,047 \\
\hline
\end{tabular}

\section{Orientação à Inovação}

(Nós somos mais inovadores do que nossos concorrentes...)

Ao decidir que métodos usar para alcançar nossas metas e objetivos.

Ao iniciar novos procedimentos ou sistemas.

Ao desenvolver novos meios para alcançar nossas metas e objetivos.

Ao promover mudanças nos conteúdos e métodos de trabalho de nossos funcionários.

$3,74 \quad 0,88 \quad 0,667 \quad 12,12$

\begin{tabular}{|c|c|c|c|c|}
\hline \multicolumn{5}{|l|}{ Habilidade de Desenvolvimento de Novos Produtos } \\
\hline $\begin{array}{l}\text { Nós temos forte vantagem competitiva sobre os } \\
\text { concorrentes nos processos eficientes para o } \\
\text { desenvolvimento de novos produtos / serviços. }\end{array}$ & 3,45 & 0,85 & 0,73 & \\
\hline $\begin{array}{l}\text { Nós temos forte vantagem competitiva sobre os } \\
\text { concorrentes na habilidade em lançar novos produtos } \\
\text { de sucesso. }\end{array}$ & 3,48 & 0,99 & 0,76 & 10,566 \\
\hline $\begin{array}{l}\text { Nós desenvolvemos ativamente novos produtos e } \\
\text { serviços para liderar o mercado. }\end{array}$ & 3,76 & 1,02 & 0,658 & 9,469 \\
\hline \multicolumn{5}{|l|}{ Sucesso de Novos Produtos } \\
\hline $\begin{array}{l}\text { Pioneirismo no mercado com novos produtos e } \\
\text { serviços. }\end{array}$ & 3,52 & 0,85 & 0,74 & \\
\hline Grau de sucesso de novos produtos. & 3,46 & 0,91 & 0,904 & 14,294 \\
\hline Taxa de introdução de novos produtos. & 3,40 & 0,90 & 0,871 & 14,076 \\
\hline \multicolumn{5}{|l|}{ Turbulência Tecnológica } \\
\hline As mudanças tecnológicas neste setor são rápidas. & 3,48 & 1,15 & - & - \\
\hline
\end{tabular}

Nota: Todas as cargas fatoriais são significantes $(p<0.001)$.

Fonte: dados da pesquisa 


\section{RESULTADOS}

As hipóteses relativas aos antecedentes da HDNP foram testadas através da análise de regressão linear e a hipótese de moderação foi testada com análise de regressão hierárquica. Os termos de interação, para a regressão hierárquica, foram criados por meio do mean centred das variáveis independentes para reduzir a multicolinealidade (AIKEN; WEST, 1991). Os resultados são apresentados nas tabelas 3 e 4 .

A tabela 3 mostra o impacto dos antecedentes da HDNP. Nota-se que a orientação à inovação exerce o maior efeito positivo sobre a $\operatorname{HDNP}(\beta=0,387 ; p<0,01)$, seguida da orientação ao consumidor $(\beta=0,158 ; p<0,01)$ e da orientação para aprendizagem $(\beta=0,131 ; p<0,05)$. Esses resultados confirmam as hipóteses $\mathrm{H} 1, \mathrm{H} 2$ e $\mathrm{H} 3$.

Tabela 3: Coeficientes do Modelo Estrutural

\begin{tabular}{|c|c|c|}
\hline \multirow[t]{2}{*}{ Path } & \multicolumn{2}{|c|}{ Modelo } \\
\hline & $\begin{array}{c}\text { STD } \\
\text { Coefficient }\end{array}$ & $\begin{array}{c}t \\
\text { value }\end{array}$ \\
\hline \multicolumn{3}{|l|}{ Efeitos Principais } \\
\hline $\begin{array}{l}\text { H1: Orientação ao Consumidor } \rightarrow \text { Habilidade de Desenvolvimento de Novos } \\
\text { Produtos }\end{array}$ & $0,158^{* *}$ & 2,593 \\
\hline $\begin{array}{l}\text { H2: Orientação à Aprendizagem } \rightarrow \text { Habilidade de Desenvolvimento de Novos } \\
\text { Produtos }\end{array}$ & $0,131^{*}$ & 2,082 \\
\hline $\begin{array}{l}\text { H3: Orientação à Inovação } \rightarrow \text { Habilidade de Desenvolvimento de Novos } \\
\text { Produtos }\end{array}$ & $0,387^{* *}$ & 6,734 \\
\hline \multicolumn{3}{|l|}{ Variáveis de Controle } \\
\hline Número de funcionários &,- 002 & $-0,035$ \\
\hline Mudanças nos desejos dos clientes & ,073 & 1,371 \\
\hline Nível de concorrência &, 022 & 0,427 \\
\hline $\begin{array}{l}R^{2} \\
\text { F Value }\end{array}$ & $\begin{array}{l}0,312^{\star \star} \\
19,388\end{array}$ & \\
\hline
\end{tabular}

$\mathrm{Na}$ tabela 4, o Modelo 1 descreve os efeitos principais e de controle. O Modelo 2 inclui a interação entre a HDNP e a Turbulência Tecnológica. O segundo modelo explicou 25,4\% da variável dependente - Sucesso de Novos Produtos e agregou 1,2\% a sua variância explicada. O Modelo 2 também apresentou um significativo impacto da HDNP no Sucesso de Novos Produtos $(\beta=0,475$, $\mathrm{p}<0,01)$. Este resultado oferece sustentação à $H 4$.

$O$ termo de interação também apresentou coeficientes significantes. HDNP $\times$ Turbulência Tecnológica mostrou um impacto positivo no Sucesso de Novos Produtos $(\beta=0,110, p<0,05$ ), sustentando assim H5. Neste sentido, nas empresas que convivem com uma alta turbulência tecnológica, existe uma influência mais forte da HDNP no Sucesso de Novos Produtos. 
Tabela 4 - Efeitos principais e moderador do modelo estrutural e variáveis de controle

\begin{tabular}{|c|c|c|c|c|}
\hline \multirow[t]{2}{*}{ Path } & \multicolumn{2}{|c|}{ Modelo 1} & \multicolumn{2}{|c|}{ Modelo 2} \\
\hline & $\begin{array}{c}\text { STD } \\
\text { Coefficient }\end{array}$ & $\begin{array}{c}t \\
\text { value }\end{array}$ & $\begin{array}{c}\text { STD } \\
\text { Coefficient }\end{array}$ & $\begin{array}{c}t \\
\text { value }\end{array}$ \\
\hline \multicolumn{5}{|l|}{ Efeitos Principais } \\
\hline $\begin{array}{l}\text { H4: Habilidade de Desenvolvimento de Novos Produtos } \\
\quad \rightarrow \text { Sucesso de Novos Produtos }\end{array}$ & $0,464^{* *}$ & 8,232 & $0,475^{\star *}$ & 8,433 \\
\hline $\begin{array}{l}\text { Turbulência Tecnológica } \rightarrow \text { Sucesso de Novos } \\
\quad \text { Produtos }\end{array}$ & 0,039 & 0,645 & 0,030 & 0,502 \\
\hline \multicolumn{5}{|l|}{ Efeito Moderador } \\
\hline $\begin{array}{l}\text { x Turbulência Tecnológica } \rightarrow \text { Sucesso de Novos } \\
\text { Produtos }\end{array}$ & & & $0,110^{*}$ & 1,973 \\
\hline \multicolumn{5}{|l|}{ Variáveis de Controle } \\
\hline Número de funcionários & 060 & 1,081 & 060 & 1,081 \\
\hline Mudanças nos desejos dos clientes & 061 & ,992 & 061 & ,992 \\
\hline Nível de concorrência &, 023 &, 404 & ,023 &, 404 \\
\hline$R^{2}$ Change & & & $0,012^{*}$ & \\
\hline F Value & & & 3,892 & \\
\hline$R^{2}$ & $0,242^{\star *}$ & & $0,254^{*}$ & \\
\hline F Value & 15,747 & & 13.925 & \\
\hline
\end{tabular}

Nota: ${ }^{*} p<0,05^{* *} p<0,01$

Fonte: dados da pesquisa

Com o intuito de reforçar este resultado, também foi testado o impacto da HDNP no Sucesso de Novos Produtos para grupos de empresas com alto e baixo níveis de turbulência tecnológica separadamente. Para isso, a amostra foi divida em três grupos em termos de nível de turbulência tecnológica. Os tamanhos dos subgrupos foram: 62 (baixa turbulência tecnológica) e 62 (alta turbulência tecnológica), o grupo com níveis médios de turbulência tecnológica foi desconsiderado, e então foi calculado o coeficiente de regressão da HDNP no Sucesso de Novos Produtos (variáveis de controle também foram incluídas). No grupo de baixa turbulência tecnológica, o efeito da HDNP no Sucesso de Novos Produtos foi positivo e significante $(\beta=0,318, p<0,05)$. De outra forma, no grupo de alta turbulência tecnológica, a HDNP mostrou um impacto mais forte no Sucesso de Novos Produtos $(\beta=0,572, p<0,01)$. A Figura 2 apresenta um gráfico da relação entre a HDNP e o Sucesso de Novos Produtos, com a ação do moderador Turbulência Tecnológica.

Figura 2: Representação gráfica do efeito moderador da Turbulência Tecnológica

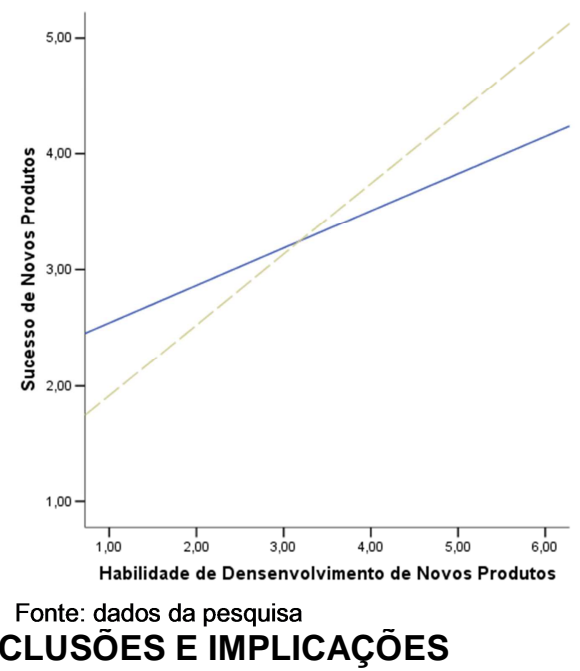

Alta Turbulência

Baixa Turbulência

5 CONCLUSŌES E IMPLICAÇŌES

Estudos do CEPE [ISSN 1982-6729]. Santa Cruz do Sul, n. 43, p. 119-135, jan./jun. 2016.

https://online.unisc.br/seer/index.php/cepe/index 
O teste empírico do modelo teórico proposto revelou sustentação para todas as hipóteses apresentadas, demonstrando assim que a Habilidade de Desenvolvimento de Novos Produtos constitui um importante antecedente do Sucesso de Novos Produtos, respondendo por $47,5 \%$ da variação na variável dependente (SNP). A HDNP, no entanto, revelou-se mais relevante quando da presença do moderador Turbulência Tecnológica. A turbulência tecnológica de um setor implica no surgimento constante de novas tecnologias que agilizam os processos internos das organizações e dinamizam as suas atividades. Assim, em alta turbulência tecnológica, as empresas precisam manterse atualizadas para sobreviver no mercado e conquistar vantagem competitiva. Nestes ambientes, quanto mais houver habilidade para lidar com estas novas tecnologias, maior será o seu sucesso com novos produtos lançados.

Por outro lado, ambientes com baixa turbulência tecnológica não exigem tanta habilidade por parte das empresas para conquistar sua fatia de mercado, pois o ritmo das mudanças é mais lento, o que oferece mais tempo de adaptação às novas tecnologias e faz com que a habilidade não seja tão decisiva para o resultado.

Neste sentido, o antecedente que exerceu maior influência na HDNP foi a Orientação à Inovação, que significa a disposição da organização para adotar novos procedimentos de trabalho. Dentre as variáveis que compuseram este construto, revelaram-se mais representativas a postura inovadora na definição de métodos para alcançar metas e objetivos e na iniciação de novos procedimentos ou sistemas (conforme Tabela 2). Este resultado vai ao encontro da importância da HDNP em ambientes com alta turbulência tecnológica, demonstrando que a abertura para adoção de novas posturas e implantação de novas tecnologias influenciam significativamente na HDNP, que conduz ao SNP.

A Orientação ao Consumidor foi o segundo antecedente mais influente na HDNP. Este construto revelou maior representatividade no que se refere à consideração das necessidades dos clientes, tanto para formação de estratégias competitivas quanto para o alinhamento das funções do negócio. Estes resultados demonstram que a HDNP está mais associada ao estudo dos desejos dos consumidores do que ao incremento do valor ou da satisfação. Assim, o contexto pesquisado revelou que as empresas estão mais atentas à captação de clientes (na medida em que estudam as necessidades do mercado e buscam meios para atendê-las) do que à manutenção de clientes (como a criação de valor e satisfação).

A Orientação à Aprendizagem foi o terceiro antecedente mais influente na HDNP, sendo que as variáveis mais importantes na formação deste construto foram a percepção do treinamento e da aprendizagem como investimos e a visão do aprendizado como fator chave para melhorias. $O$ fato da OA ter sido apontada como o antecedente de menor implicação na HDNP demonstra que no contexto estudado - empresas industriais - existe uma preocupação maior das empresas em incentivar o processo criativo e o surgimento de novas ideias e não é tão enfatizada a construção e incorporação de conhecimento internamente. Este resultado pode ser atribuído ao fato de o setor industrial ser dotado de grandes avanços tecnológicos e por isso exigir uma postura mais inovadora das empresas quando comprada à aprendizagem.

Estudos do CEPE [ISSN 1982-6729]. Santa Cruz do Sul, n. 43, p. 119-135, jan./jun. 2016. https://online.unisc.br/seer/index.php/cepe/index 
O presente estudo contribui para academia na consideração do construto Habilidade de Desenvolvimento de Novos Produtos, que é pouco explorado na literatura. Assim, o estudo apresentou indicadores para mensurar a HDNP, bem como a posicionou no modelo teórico proposto, investigando os seus antecedentes e o seu efeito no Sucesso de Novos Produtos. Além disso, o estudo apresenta o construto de Turbulência Tecnológica, que frequentemente é testado em combinação com outras variáveis como a Turbulência de Mercado, contribuindo assim para a investigação de fatores específicos externos às organizações como moderadores de indicadores internos.

O presente estudo possui como limitações o fato de ter considerado apenas empresas brasileiras do ramo industrial, bem como pela amostra de respondentes ser composta por apenas um profissional de cada empresa pesquisada. Neste sentido, sugere-se que estudos futuros busquem ampliar o escopo desta pesquisa, considerando outros setores do mercado, bem como outros profissionais envolvidos com o processo de desenvolvimento de novos produtos (não limitado aos CEOs).

\section{REFERÊNCIAS}

AIKEN, L. S.; WEST, S. G. Multiple Regression: Testing and Interpreting Interactions. Newbury Park, CA: Sage Publications, 1991.

AKGÜN, A. E.; KESKIN, H.; BYRNE, J. Antecedents and Contingent Effects of Organizational Adaptive Capability on Firm Product Innovativeness. Journal of Product Innovation Management, 29, 171-189, 2012.

AKMAN, G.; YILMAZ, C. Innovative capability, innovation strategy and market orientation: an empirical analysis in Turkish software industry. International Journal of Innovation Management, 12, 69-111, 2008.

ATUAHENE-GIMA, K. Resolving the Capability-Rigidity Paradox in New Product Innovation. Journal of Marketing, 69(4), 61-83, 2005.

ATUAHENE-GIMA, K.; SLATER, S. F.; OLSON, E. M. The Contingent Value of Responsive and Proactive Market Orientations for New Product Program Performance. Journal of Product Innovation Management, 22(6), 464-482, 2005.

ATUAHENE-GIMA, K.; WEI, Y. S. The Vital Role of Problem-Solving Competence in New Product Success. Journal of Product Innovation Management, 28, 81-98, 2010.

AUGUSTO, M.; COELHO, F. Market orientation and new-to-the-world products: Exploring the moderating effects of innovativeness, competitive strength, and environmental forces. Industrial Marketing Management, 38(1), 94-108, 2009.

AUH, S; MENGUC, B. The influence of top management team functional diversity on strategic orientations: The moderating role of environmental turbulence and inter-functional coordination. International Journal of Research in Marketing, 22, 333-350, 2005.

BAKER, W. E.; SINKULA, J. M. Does Market Orientation Facilitate Balanced Innovation Programs? An Organizational Learning Perspective. Journal of Product Innovation Management, 24(4), 316-334, 2007.

BAKER, W. E.; SINKULA, J. M. The Synergistic Effect of Market Orientation and Learning Orientation on Organizational Performance. Journal of the Academy of Marketing Science, v. 27, n.24, p. 411427, 1999. 
BARCZAK, G., SULTAN, F., HULTINK, E. J. Determinants of IT Usage and New Product Performance._Journal of Product Innovation Management, n.24, p. 600-613, 2007.

BRENTANI, U., KLEINSCHMIDT, E. J. Corporate Culture and Commitment: Impact on Performance of International New Product Development Programs. Journal of Product Innovation Management,_n.21, p. 309-333, 2004.

BUGANZA, T.; DELL'ERA, C.; VERGANTI, R. Exploring the Relationships Between Product Development and Environmental Turbulence: The Case of Mobile TLC Services. Journal of Product Innovation Management,_n.26, p. 308-321, 2009.

CALANTONE, R.J.; CAVUSGIL, S.T.; ZHAO, Y. Learning orientation, firm innovation capability, and firm performance, Industrial Marketing Management, 31(6), 515-524, 2002.

CALANTONE, R. J.; CHAN, K.; CUI, A. S. Decomposing Product Innovativeness and Its Effects on New Product Success. Journal of Product Innovation Management, n.23, p. 408-421, 2006.

CALANTONE, R.; GARCIA, R.; DRÖGE, C. The Effects of Environmental Turbulence on New Product Development Strategy Planning. Journal of Product Innovation Management, n.20, p. 90-103, 2003.

CARBONELL, P.; RODRÍGUEZ-ESCUDERO, A. I.; PUJARI, D. Customer Involvement in New Service Development: An Examination of Antecedents and Outcomes. Journal of Product Innovation Management, n.26, p. 536-550, 2009.

CITRIN, A. V.; LEE, R. P.; McCULLOUGH, J. Information Use and New Product Outcomes: The Contingent Role of Strategy Type. Journal of Product Innovation Management, n.24, p. 259-273, 2007.

DAY, G. S. Continuous Learning About Markets. California Management Review, 36(4), 9-31, 1994.

DAYAN, M.; ELBANNA, S. Antecedents of team intuition and its impact on the success of new product development projects. Journal of Product Innovation Management, 28, 159-174, 2011.

DESHPANDÉ, R.; FARLEY, J. U.; WEBSTER JR., F. E. Corporate Culture, Customer Orientation, and Innovativeness in Japanese firms: A Quadrad Analysis. Journal of Marketing, vol. 57, p. 23-37, 1993.

DILLON, W. R., MADDEN, T. J.; FIRTLE, N. H. Marketing Research in a Marketing Environment, St. Louis: Times Mirror, 1994.

DRÖGE, C.; CLAYCOMB, C.; GERMAIN, R. Does Knowledge Mediate the Effect of Context on Performance? Some initial evidence. Decision Science, vol. 34, n. 3, p. 541-568, 2003.

FORNELL, C.; LARCKER, D. F. Evaluating Structural Equation Models with Unobservable Variables and Measurement Error. Journal of Marketing Research, 18(1), 39-51, 1981.

GARCIA, R.; CALANTONE, R. A critical look at technological innovation typology and innovativeness terminology: a literature review. Journal of Product Innovation Management, 19, 110-132, 2002.

HOOLEY, G. J.; GREENLEY, G. E.; CADOGAN, J. W.; AND FAHY, J. The performance impact of marketing resources. Journal of Business Research, 58(1), 18-27, 2005.

HOOLEY, G. J.; MÖ LLER, K.; BRODERICK, A. J. Competitive positioning and the resource-based view of the firm. Journal of Strategic Marketing, 6(2), 97-115, 1998.

HULLAND, J. Use of Partial Least Squares (PLS) in Strategic Management Research: A Review of Four Recent Studies. Strategic Management Journal, 20(2), 195-202, 1999.

HULT, G.T.M.; HURLEY, R. F.; KNIGHT, G. A. Innovativeness: Its antecedents and impact on business performance. Industrial Marketing Management, 33, 429-438, 2004.

HURLEY, R. F.; HULT, G. T. M. Innovation, market orientation, and organizational learning: an integration and empirical examination. Journal of Marketing, 62(3), 42-54, 1998.

JAWORSKI, B. J.; KOHLI, A. K. Market Orientation: Antecedents and Consequences. Journal of Marketing, 57(3), 53-70, 1993.

KLEINSCHMIDT, E. J., BRENTANI, U., SALOMO, S. Performance of Global New Product Development Programs: A Resource-Based View. Journal of Product Innovation Management, n.24, p. 419-441, 2007. 
KOZLENKOVA, I. V.; SAMAHA, S. A.; PALMATIER, R. W. Resource-based theory in marketing. Journal of the Academy Marketing. Science, 2013.

KUIVALAINEN, O.; SUNDQVIST, S.; PUUMALAINEN, K.; CADOGAN, J. W. The Effect of Environmental Turbulence and Leader Characteristics on International Performance: Are KnowledgeBased Firms Different? Canadian Journal of Administrative Sciences, 21, 1, 35-50, 2004.

LANGERAK, F., HULTING, E. J., ROBBEN, H. S. J. The Impact of Market Orientation, Product Advantage, and Launch Proficiency on New Product Performance and Organizational Performance. Journal of Product Innovation Management,_n.21, p. 79-94, 2004.

LEDWITH, A.; O'DWYER, M. Market Orientation, NPD Performance, and Organizational Performance in Small Firms. Journal of Product Innovation Management, n.26, p. 652-661, 2009.

LEENDERS, R.J., ENGELEN, Jo M. L. van, KRATZER, J. Systematic Design Methods and the Creative Performance of New Product Teams: Do They Contradict or Complement Each Other? Journal of Product Innovation Management, n.24, p. 166-179, 2007.

LI, T.; CALANTONE, R. The Impact of Market Knowledge Competence on New Product Advantage: Conceptualization and Empirical Examination. Journal of Marketing, 62(4), p.13-29, 1998.

LUKAS, B. A.; FERRELL, O. C. The effect of market orientation on product innovation. Journal of the Academy of Market Science, 28(2), 239-247, 2000.

MACINTOSH, G. Customer orientation, relationship quality, and relational benefits to the firm. Journal of Services Marketing, 21, 150-159, 2007.

MARSH, S. J.; STOCK, G. N. Creating Dynamic Capability: The Role of Intertemporal Integration, Knowledge Retention, and Interpretation. Journal of Product Innovation Management, n.23, p. 422436, 2006.

MENGUC, B. S.; AUH, S. Development and return on execution of product innovation capabilities: The role of organizational structure. Industrial Marketing Management, 39, 820-831, 2009.

MENON, A.; BHARADWAJ, S. G.; HOWELL, R. D. The Quality and Effectiveness of Marketing Strategy: Effect of Functional and Dysfunctional Conflict in Intraorganizational Relationships. Journal of Academy of Marketing Science, 24(4), 299-313, 1996.

NAKATA, C., IM, S. Spurring Cross-Functional Integration for Higher New Product Performance: A Group Effectiveness Perspective. Journal of Product Innovation Management,_n.27, p. 554-571, 2010. NARVER, J. C.; SLATER, S. F. The Effect of a Market Orientation on Business Performance. Journal of Marketing, 54(4), 20-35, 1990.

NARVER, J. C.; SLATER, S. F.; MacLACHLAN, D. L. Responsive and Proactive Market Orientation and New-Product Success. Journal of Product Innovation Management, n.21, p. 334-347, 2004.

NGO, L. V.; O'CASS, A. In Search of Innovation and Customer-related Performance Superiority: The Role of Market Orientation, Marketing Capability, and Innovation Capability Interactions. Journal of Product Innovation Management, 29, 861-877, 2012.

PODSAKOFF, P.; MACKENZIE, S.; LEE, J. Y.; PODSAKOFF, N. Common method biases in behavioral research: A critical review of the literature and recommended remedies. Journal of Applied Psychology, 88(5), 879-903, 2003.

REID, S. E., BRENTANI, U. Market Vision and Market Visioning Competence: Impact on Early Performance for Radically New, High-Tech Products. Journal of Product Innovation Management, n.27, p. 500-518, 2010.

RHEE, J.; PARK, T.; LEE, D. H. Drivers of innovativeness and performance for innovative SMEs in South Korea: Mediation of learning orientation. Technovation, 30(1), 65-75, 2010.

RIJSDIJK, S. A.; LANGERAK, F.; HULTINK, E. J. Understanding a Two-Sided Coin: Antecedents and Consequences of a Decomposed Product Advantage. Journal of Product Innovation Management, 28, 33-47, 2010. 
SALOMO, S.; TALKE, K.; STRECKER, N. Innovation Field Orientation and Its Effect on Innovativeness and Firm Performance. Journal of Product Innovation Management,_n.25, p. 560-576, 2008.

SIGUAW, J. A.; SIMPSON, P. N.; ENZ, C. A. Conceptualizing Innovation Orientation: A Framework for Study and Integration of Innovation Research. Journal of Product Innovation Management,_n.23, p. 556-574, 2006.

SINKULA, J. M.; BAKER, W. E.; NOORDEWIER, T. A Framework for Market-Based Organizational Learning: Linking Values, Knowledge and Behavior. Journal of the Academy of Marketing Science, 25(4), 305-318, 1997.

SNOJ, B.; MILFELNER, B.; GABRIJAN, V. An Examination of the Relationships among Market Orientation, Innovation Resources, Reputational Resources, and Company Performance in the Transitional Economy of Slovenia. Canadian Journal of Administrative Sciences, n.24, P. 151-164, 2007.

SPANJOL, J., QUALLS, W. J.; ROSA, J. A. How Many and What Kind? The Role of Strategic Orientation in New Product Ideation. Journal of Product Innovation Management, 28, 236-250, 2011.

STOCK, R. M.; ZACHARIAS, N. A. Patterns and performance outcomes of innovation orientation. Journal of the Academy Marketing Science, 39, 870-888, 2011.

SUBRAMANIAM, M.; YOUNDT, M. A. The influence of intellectual capital on the types of innovative capabilities. Academy of Management Journal, 48, 450-463, 2005.

TALKE, K., HULTINK, E. J. The Impact of the Corporate Mind-set on New Product Launch Strategy and Market Performance. Journal of Product Innovation Management, n.27, p. 220-237, 2010.

TAKE, K.; SALOMO, S.; KOCK, A. Top Management Team Diversity and Strategic Innovation Orientation: The Relationship and Consequences for Innovativeness and Performance. Journal of Product Innovation Management, n.28, p. 819-832, 2011.

VAN DE VEN, A. Central problems in the management of innovation. Management Science, v. 32, n. $5,1986$.

VORHIES, D. W.; MORGAN, R. E.; AUTRY, C. W. Product-market strategy and the marketing capabilities of the firm: impact on market effectiveness and cash flow performance. Strategic Management Journal, 30, 1310-1334, 2009.

WEI, Y.; ATUAHENE-GIMA, K. The moderating role of reward systems in the relationship between market orientation and new product performance in China. International Journal of Research in Marketing, 26(2), 89-96, 2009.

WEI, Y. (Susan), MORGAN, N. A. Supportiveness of Organizational Climate, Market Orientation, and New Product Performance in Chinese Firms. Journal of Product Innovation Management, n.21, p. 375-388, 2004.

WEST, M.A.; ANDERSON, N.R. Innovation in top management team. Journal of Applied Psychology, 81(6), 680-693, 1996.

ZHOU, K. Z., YIM, B. C. K., TSE, D. K. C. The Effects of Strategic Orientations on Technology-and Market-Based Breakthrough Innovations'. Journal of Marketing, v. 69, n. 2, p. 42-60, 2005.

ZOU, S.; ANDRUS, D.; NORVELL, D. Standardization of international marketing strategy by firms from a developing country. International Marketing 